\title{
FORMACIÓN POR COMPETENCIAS EN EDUCACIÓN SUPERIOR. UNA APROXIMACIÓN CONCEPTUAL A PROPÓSITO DEL CASO COLOMBIANO
}

Walter Alfredo Salas Zapata

Universidad de Antioquia, Colombia

\section{INTRODUCCIÓN}

Ante la implementación de los exámenes de estado en Colombia para evaluar la calidad de la educación superior, el tema de las competencias toma una relevancia particular. Naturalmente se podría pensar que una evaluación por competencias necesariamente se deriva de un modelo de formación por competencias, pero ese no ha sido el caso colombiano, lo que ha obligado a académicos y pedagogos a repensar la educación de los estudiantes bajo un modelo de formación por competencias.

En esa reflexión alrededor de las competencias surgen preguntas como ¿qué tipo de sujeto estamos formando?, ¿cómo trasciende un modelo de formación por competencias el proceso de aprendizaje?, ¿qué implicaciones conlleva la implementación de un modelo de formación por competencias? y ¿es éste un modelo ideal y a la vez factual?, si el fin es lograr la formación de un mejor sujeto para la sociedad ¿Cuál es el aporte de las competencias a dicha formación?

He aquí una aproximación conceptual al modelo de formación por competencias, que si bien no da respuesta a todas las preguntas que se derivan de este tema, se constituye en un insumo generador de inquietudes en torno al tema.

\section{EL CONTEXTO DE LA EDUCACIÓN.}

En la era de la globalización las sociedades han experimentado grandes cambios en los diferentes aspectos de la vida humana. Desde una perspectiva económica podemos afirmar que en el marco de la "mundialización" del neoliberalismo, el capitalismo ha atravesado las fronteras internacionales por medio de la eliminación de las barreras arancelarias y la realización, cada vez con más frecuencia, de transacciones transnacionales y la apertura de los mercados al libre comercio.

En una dimensión política hemos visto como los países cada vez más se fijan objetivos comunes en defensa de la democracia, la seguridad y la protección del medio ambiente mediante la firma de tratados y acuerdos internacionales logrados en las cumbres y reuniones de jefes de estado o de sus delegados.

De otro lado, son claros los cambios sociales que ha traído la llegada de la sociedad post-industrial, mas conocida como sociedad de la información que, con el avance tecnológico logrado en las telecomunicaciones, la informática, los medios de transporte y en la industria en general, ha convertido las sociedades cerradas en sociedades abiertas a la llamada aldea global.

La producción excesiva de información y el transporte de la misma mediante los sistemas de información a través del mundo a velocidades sin precedentes, exige del ser humano una mayor capacidad de adaptación al medio, reflejada en la autonomía requerida para llevar a cabo los procesos que hacen 
parte de la cotidianidad. Exige autonomía en el trabajo, autonomía para pensar y, en el marco de la educación, autonomía para aprender.

\section{ANTECEDENTES DE LOS PARADIGMAS EDUCATIVOS EN COLOMBIA.}

Al dar una mirada a lo que ha sido la educación en Colombia se puede ver cómo los procesos de formación se han caracterizados por estar centrados en la enseñanza más que en el aprendizaje. No obstante, al igual que en otros países, existe una tendencia a señalar el aprendizaje como objetivo fundamental de los procesos de formación.

En Europa, ello se ha logrado a través de grandes reformas en educación realizadas, en promedio, cada 25 años, que es el período de duración de una generación educativa (lafrancesco, 2004), en los que ha cambiado tanto la función de la educación como los roles del estudiante y el profesor.

En Colombia solamente se han realizado dos reformas estructurales del sistema educativo. La primera cuando por primera vez se organiza la educación bajo la responsabilidad del Ministerio de Instrucción Pública, con la ley 39 de 1903 sobre la Instrucción Pública, en la que no se define qué es la educación ni mucho menos sus objetivos. La segunda gran reforma se realizó con la ley 115 de 1994 en la que se define la educación como un proceso de formación permanente, personal, cultural y social. De esto se infiere que en Colombia se trasladó la visión de la educación como un proceso de instrucción a uno de formación del sujeto en un contexto social y cultural.

Si se divide el siglo XX en períodos de 25 años (lbid) se vislumbra entonces que entre 1903 y 1925 la función de la educación fue instruir, por consiguiente la función del profesor era "decir" y la del alumno era "oír". En el siguiente período el profesor tomó el nombre de maestro en tanto que su función era "explicar", mientras el alumno tomó el nombre de estudiante y ya su función era "entender". Cerca de 1950 los llamados docentes, en el marco del proceso educativo deben "demostrar" a sus discentes mediante la "experimentación". No obstante, alrededor de 1975, con el surgimiento de las corrientes constructivitas, la función de los educandos era ya la de "aprender"a través de un proceso de construcción del conocimiento acompañado por el educador.

Finalmente, hacia el año 2000 surge un modelo en el que el educador cumple con el rol de mediador en el proceso de formación que busca hacer del educando un líder agente de transformación competitivo en la sociedad. No obstante, para competir es necesario actuar y conocer el medio en el que se compite, por ello ese proceso de formación de sujetos tiene como propósito la transformación de la sociedad a través de la solución de problemas por parte de un individuo que la conoce, que se conoce a sí mismo, que conoce el problema, su abordaje conceptual y factual y que aprende cuál es la mejor manera de traducir los conceptos en hechos.

La tendencia que ha seguido la educación en el último siglo ha sido el otorgarle cada vez mayor protagonismo al estudiante en su proceso de formación. Por ello el hecho de pretender que el estudiante conozca el medio, se conozca a sí mismo, conozca los conocimientos y la manera mas adecuada para llegar a ellos; implica todo un proceso de aprendizaje autónomo en el que él aprenda a aprender; siendo éste un requisito para la formación por competencias. 
Figura 1.

Antecedentes de la educación. (Tomado de lafrancesco, 2004)

\begin{tabular}{|c|c|c|c|c|}
\hline \multicolumn{5}{|c|}{ ANTECEDENTES EN LA EDUCACIÓN } \\
\hline & $\begin{array}{l}\text { Nombre del } \\
\text { acompañante }\end{array}$ & Función & $\begin{array}{l}\text { Nombre del sujeto } \\
\text { de la educación }\end{array}$ & Funcion \\
\hline 1903 & Profesor & Decir & Alumno & Oir \\
\hline 1925 & Maestro & Explicar & Estudiante & Entender \\
\hline 1950 & Docente & Demostrar & Discente & Experimentar \\
\hline 1975 & Educador & Construir & Educando & Aprender \\
\hline 2000 & Mediador & Transformar & Lider Transf. & Competir \\
\hline & & $\overline{\text { Ense }}$ & $\underset{\text { r a aprender }}{\longrightarrow}$ Aprendi & \\
\hline
\end{tabular}

\section{EL PROCESO DE APRENDIZAJE}

El aprendizaje se concibe como la reconstrucción de los esquemas de conocimiento del sujeto a partir de las experiencias que éste tiene con los objetos -interactividad- y con las personas intersubjetividad - en situaciones de interacción que sean significativas de acuerdo con su nivel de desarrollo y los contextos sociales que le dan sentido (Segura, 2003).

El proceso de aprendizaje concebido desde la perspectiva constructivista de Ausubel, es el proceso por el cual el sujeto del aprendizaje procesa la información de manera sistemática y organizada y no solo de manera memorística sino que construye conocimiento (Díaz, 1998:18). En este proceso se pueden identificar claramente tres factores que son determinantes en el aprendizaje (lafrancesco, 2004), como son las actitudes, las aptitudes y los contenidos. No obstante, a partir de las investigaciones de Piaget dichas aptitudes toman dos orientaciones diferentes, las aptitudes intelectivas y las aptitudes procedimentales.

El desarrollo de cada una de las actitudes, aptitudes intelectivas, aptitudes procedimentales y los contenidos tiene correspondencia con la formación en el ser, en el pensar, el hacer y el saber, respectivamente, y el aprendizaje logrado por medio de la convergencia de estas cuatro dimensiones da lugar a los llamados aprendizajes significativos, que son los aprendizajes en los cuales el sujeto del proceso de formación reconfigura la información nueva con la experiencia, permitiéndole así integrar grandes cuerpos de conocimiento con sentido. De esa integración entre conocimiento con sentido y experiencia resulta el desarrollo de la competencia (Ibíd.). 
Figura 2.

Factores que intervienen en el proceso de aprendizaje

\section{Factores que intervienen en el aprendizaje}

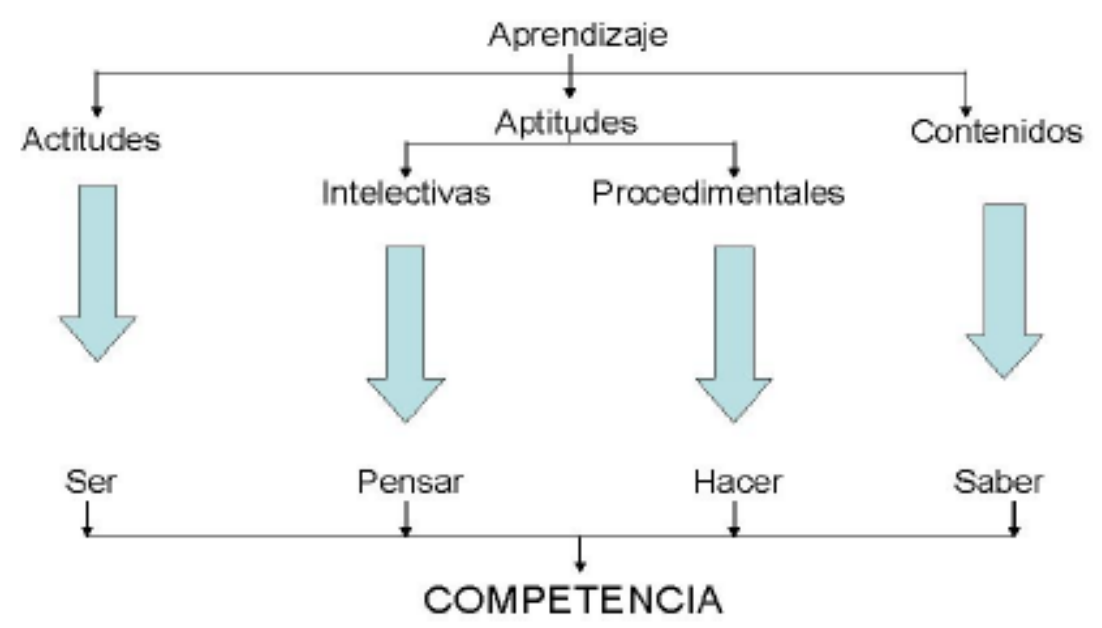

\section{FACTORES QUE INTERVIENEN EN EL PROCESO DE APRENDIZAJE.}

Es necesario dar una mirada inicial a los factores que intervienen en el proceso de aprendizaje para luego discernir acerca de cómo ellos contribuyen al desarrollo de las competencias. Se definen estos cuatro factores (Ibíd.), así:

\section{A La actitud}

Es una predisposición afectiva y motivacional requerida para el desarrollo de una determinada acción, posee también un componente cognitivo y un componente comportamental.

En la actitud lo fundamental es generar expectativa, porque así el estudiante se interesa y se motiva en su proceso de aprendizaje. No obstante la actitud puede ser inversamente proporcional a la aptitud por un mecanismo de compensación de debilidades, como en el caso de quienes al reconocer sus debilidades en el área de matemáticas, en medio de la necesidad de aprender, se interesan más por aprender que aquellos que tienen más habilidades para dicha área.

\section{B. Aptitudes intelectivas}

Son habilidades mentales que determinan el potencial de aprendizaje, también definidas como las capacidades para pensar y saber (lafrancesco, 2004). Dependen de la estructura mental, las funciones cognitivas, los procesos de pensamiento y las inteligencias múltiples.

\section{Aptitudes procedimentales}

Se definen como las capacidades para actuar y hacer (Ibid.) Están relacionadas con los métodos, técnicas, procesos y estrategias empleadas en el desempeño.

\section{Contenidos}

Es toda la estructura conceptual susceptible de ser aprendida. Su organización es vital para el proceso de aprendizaje. En la medida en que exista más coherencia entre ellos, los estudiantes encontrarán las relaciones entre los mismos lo que a su vez aumentará su nivel de compresión. La comprensión de los conceptos determina el aprendizaje, mas no el aprendizaje significativo. De ahí decimos que quién sabe 
actuar, y lo hace bien porque además del dominio conceptual, comprende como funciona su pensamiento y como se interrelacionan los conceptos en ese proceso de aprendizaje, ha desarrollado la competencia. No obstante es importante ilustrar al lector con algunos conceptos de competencias.

\section{COMPETENCIAS}

En el momento de definir las competencias es difícil tomar como referente un solo concepto, pues son tan variadas y acertadas las definiciones que referirse solo a una representaría un sesgo para un completo abordaje del concepto de competencias desde la complejidad que él exige.

De ahí que la competencia puede definirse de manera sencilla como "el resultado de un proceso de integración de habilidades y de conocimientos; saber, saber-hacer, saber-ser, saber-emprender..." (Chavez, 1998). No obstante esta definición no deja entrever el papel fundamental que cumple el contexto cultural en el desarrollo de las competencias.

Si nos remitimos al concepto original de competencias es inevitable retomar el enfoque de Noam Chomsky quién, a partir de su fascinación por el proceso de apropiación que hace el niño del sistema de la lengua y de esa capacidad extraordinaria y misteriosa para interiorizar el mundo, en la búsqueda de la elaboración de una teoría sobre el origen y dominio del lenguaje, introduce el concepto de competencia y de actuación.

Desde la perspectiva lingüística de Chomsky se define la competencia como el dominio de los principios que gobiernan el lenguaje; y la actuación como la manifestación de las reglas que subyacen al uso del lenguaje (Trujillo, 2001). Por ello a partir de Chomsky surge el concepto de competencias como el de dominio de los principios: capacidad, y la manifestación de los mismos, actuación o puesta en escena.

Un enfoque similar es el de Piaget, quien a diferencia de Chomsky postula que esas reglas y principios están subordinadas a una lógica de funcionamiento particular, y no a una lógica de funcionamiento común. No obstante, los dos coinciden en ver la competencia como un conocimiento actuado de carácter abstracto, universal e idealizado con una considerable independencia del contexto. Desde esta lógica el conocimiento es de carácter independiente del contexto pero la actuación se enmarca en un sistema de conocimientos y es ahí donde se empieza a hablar de competencias cognitivas (Torrado, 2000).

Por su parte Hymes, desde la teoría sociolingüística considera que en el desarrollo de la competencia es el conocimiento el que se adecua a todo un sistema social y cultural que le exige utilizarlo apropiadamente. En esa misma línea Vigotsky propone que el desarrollo cognitivo, mas que derivarse del despliegue de mecanismos internos, resulta del impacto que tiene la cultura sobre el individuo en la realización de las funciones sicológicas, como en el caso del lenguaje. Por ello la competencia puede entenderse como "capacidad de realización, situada y afectada por y en el contexto en que se desenvuelve el sujeto" (Ibid).

La competencia también puede ser entendida como una "actuación idónea que emerge de una tarea concreta, en un contexto con sentido" (Bogoya, 2000), por lo tanto exige del individuo la suficiente apropiación de un conocimiento para la resolución de problemas con diversas soluciones y de manera pertinente, por ello la competencia se desarrolla en una situación o contexto determinado. 


\section{SABER HACER EN CONTEXTO...}

Ante la implementación de los exámenes de calidad en educación superior en Colombia, el Instituto Colombiano para el Fomento de la Educación Superior -ICFES- como institución encargada de emitir directrices en evaluación por competencias para la educación superior, define las competencias como un conjunto de acciones que el sujeto realiza cuando interactúa significativamente en un contexto determinado, definición que se resume en: un saber hacer en contexto... (ICFES, 1999).

$\mathrm{Si}$ bien las definiciones que hemos visto difieren en algunos aspectos, al tomar sus puntos de convergencia se pueden definir las competencias como un saber hacer en un contexto dinámico de un sujeto con capacidad de creatividad, adaptación y asimilación de lo nuevo, en situaciones concretas, lo que en última instancia se reduce a "sujeto que idóneamente resuelve algo preciso" (Marín, 2002).

Pero si en estas definiciones de competencias los autores tienen puntos de encuentro en dos elementos fundamentales como son el saber y el contexto, en lo que no hay claridad es en la definición de contexto, pues desde la perspectiva piagetiana el contexto tomado como un sistema de conocimientos no sería otra cosa que el saber disciplinar, y de ahí el desarrollo de las competencias cognitivas. Pero desde la mirada vigotskyana, en la que se concibe el contexto como el entorno cultural y la cotidianidad que hay inmersa en él, se desarrollarían competencias de otro tipo, como las comunicativas por ejemplo.

Éste es un aspecto que no queda muy claro con la definición "saber hacer en contexto" que emite el ICFES, pero que en el momento de determinar el tipo de competencias a evaluar sí, pues en el examen de estado las competencias se circunscribirán a las acciones de tipo interpretativo, argumentativo y propositivo... propias de los contextos disciplinares (ICFES, 1999).

Es necesario aclarar entonces que el contexto demanda del individuo exigencias de diverso orden como de lo cognitivo, comunicativo, axiológico, estético, etc. Exigencias que son propias del entorno cultural en donde el sujeto para interactuar con él requiere desarrollar dichas competencias.

\section{TIPOS DE COMPETENCIAS}

Aun cuando la clasificación de las competencias es muy diversa y también depende del interés del autor, es necesario tomar el enfoque del ICFES, ya que es esta la institución encargada de la realización de los Exámenes de Calidad en Educación Superior (ECAES), prueba de evaluación por competencias.

Para tales efectos el ICFES establece tres tipos de competencias que los estudiantes deben desarrollar y que por consiguiente son susceptibles de ser evaluadas: 
Tabla 1.

Competencias evaluadas por el ICFES. (Tomado de Segura, 2003)

\begin{tabular}{|c|c|c|}
\hline Tipos & Descripción & Acciones Especificas \\
\hline 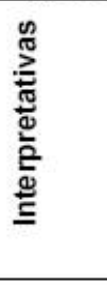 & $\begin{array}{l}\text { Comprensión de } \\
\text { información en } \\
\text { cualquier sistema } \\
\text { de símbolos o } \\
\text { formas de } \\
\text { representación }\end{array}$ & $\begin{array}{l}\text { Interpretar textos: } \\
\text { Comprender proposiciones y párrafos. } \\
\text { Identificar argumentos, ejemplos, } \\
\text { contraejemplos y demostraciones. } \\
\text { Comprender problemas. } \\
\text { Interpretar cuadros, tablas, gráficos, } \\
\text { diagramas, dibujos y esquemas. } \\
\text { Interpretar mapas, planos y modelos. }\end{array}$ \\
\hline 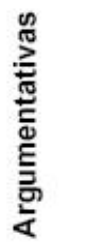 & $\begin{array}{l}\text { Explicación y } \\
\text { justificación de } \\
\text { enunciados y } \\
\text { acciones. }\end{array}$ & $\begin{array}{l}\text { Explicar el por qué, cómo y para qué. } \\
\text { Demostrar hipótesis. } \\
\text { Comprobar hechos. } \\
\text { Presentar ejemplos y contraejemplos. } \\
\text { Articular conceptos. } \\
\text { Sustentar conclusiones. }\end{array}$ \\
\hline 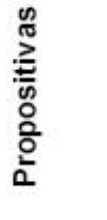 & $\begin{array}{l}\text { Producción y } \\
\text { creación }\end{array}$ & $\begin{array}{l}\text { Plantear y resolver problemas. } \\
\text { Formular proyectos. } \\
\text { Generar hipótesis. } \\
\text { Descubrir regularidades. } \\
\text { Hacer generalizaciones. } \\
\text { Construir modelos. }\end{array}$ \\
\hline
\end{tabular}

\section{FORMACIÓN POR COMPETENCIAS Y ALGUNAS IMPLICACIONES}

Ante la implementación de una evaluación por competencias para la educación superior en Colombia es necesario hacer una reflexión alrededor de un tipo de evaluación de una educación derivada de un modelo de formación que aun no hemos establecido en nuestras universidades, la formación por competencias. Ello se constituye en una razón para repensar la educación superior en Colombia, y considerar las implicaciones curriculares, didácticas y evaluativas (Gómez, 2002) que ello acarrea, y que por consiguiente obliga a replantearla desde el currículo, la didáctica y la evaluación

\section{Implicaciones curriculares}

Según Gómez, la primera implicación curricular es la revisión de los propósitos de formación del currículo; su respuesta lleva necesariamente a una evaluación de la pertinencia del mismo, y se constituye en el insumo requerido para replantear la organización de los contenidos del plan de estudios, dada tradicionalmente en asignaturas o materias.

Diseñar un currículo por competencias implica construirlo sobre núcleos problemáticos al que se integran varias disciplinas, currículo integrado, y se trabaja sobre procesos y no sobre contenidos; por ejemplo, los educandos ya no tienen que estudiar los cursos de biología y química para comprender la organización y el funcionamiento celular, sino estudiar un curso de organización y funcionamiento celular al que se integran las disciplinas apropiadas de la biología y la química, pues lo importante es la comprensión del proceso biológico y no la acumulación de todos los conocimientos de la biología.

\section{Implicaciones didácticas}

A nivel didáctico Gómez propone a la docencia el cambio de metodologías transmisionistas a metodologías centradas en el estudiante y en el proceso de aprendizaje. Un buen ejemplo de ello lo 
constituyen las metodologías activas como el Seminario Investigativo Alemán, el Aprendizaje Basado en Problemas (ABP) y el Modelo Didáctico Operativo, entre otros.

No obstante, también advierte sobre el riesgo de que los estudiantes se dispersen en las diferentes actividades y por ende no perciban la coherencia y unidad en un horizonte conceptual. De ahí la importancia del dominio metodológico y de trabajar conceptos estructurales en función de dominios cognitivos donde las estrategias docentes apunten hacia la interconexión de los temas (lbid).

\section{Implicaciones en la evaluación}

La evaluación es uno de los puntos más complejos en la formación por competencias, pues una evaluación por competencias implicaría una reforma radical del sistema educativo, implica esencialmente el cambio de una evaluación por logros a una evaluación por procesos, por lo tanto no se evalúa un resultado sino todo el proceso de aprendizaje, en el que a su vez interfiere el contexto, la motivación, los sistemas simbólicos y el desarrollo cognitivo. Ello implica hacer un seguimiento al proceso de aprendizaje desde la motivación misma hasta la ejecución de la acción y su consecuente resultado.

\section{METODOLOGÍAS DE TRABAJO POR COMPETENCIAS}

Gómez propone básicamente tres metodologías para realizar trabajo por competencias. Ellas son:

- Trabajo por proyectos: En el que a partir de una situación problema se desarrollan procesos de aprendizaje y de construcción de conocimiento, vinculados al mundo exterior, a la cotidianidad $y$ al contexto.

- Resolución de problemas: Esta metodología permite hacer una activación, promoción y valoración de los procesos cognitivos cuando los problemas y tareas se diseñan creativamente. Los talleres y seminarios son un buen ejemplo de ello.

- Enseñanza para la comprensión:

Desde la perspectiva de Perkins, enfocar el proceso de aprendizaje hacia la comprensión implica organizar las imágenes y las representaciones en diferentes niveles para lograr la comprensión por parte de los estudiantes, consecuentemente ellos aprenden a comprender y por consiguiente logran conciencia sobre cómo ellos comprenden.

Comprender es el proceso por el cual se asimilan las representaciones y se les otorga un significado. Existen diversas metodologías que propician los cinco niveles de comprensión dados por Perkins (lbid):

- Narrativo. Cuando el estudiante presenta un relato o narración acerca del concepto. Ej: Un relato sobre el origen de la constitución.

- Lógico - cuantitativo. Cuando el estudiante aborda el concepto mediante procesos deductivos o numéricos. Ej: Los análisis numéricos de contraste o comparar modelos económicos.

- Fundacional. Se aborda el concepto epistemológicamente desde sus diferentes connotaciones. Ej: El concepto de independencia, clima, socialización.

- Estético. Se da un abordaje del concepto desde la vivencia. Ej: El estudio del estado a partir del folklore y la cultura. 
- Experimental. Cuando la manipulación de datos y el control de variables generan cambios conceptuales. Genera en el estudiante la capacidad propositiva y trasformativa.

\section{APUNTES FINALES}

Lo anterior es solo una propuesta metodológica de cómo abordar una tema álgido y de difícil abordaje como es el de las competencias, un tema que por su relativa juventud se considera en construcción y aun con grandes vacíos en los que cada uno se constituiría en el objeto de otros escritos. Esta mirada general al panorama ofrecido por las competencias es solo un acercamiento a los diferentes puntos de debate como lo pueden ser la evaluación, el currículo y la formación por competencias entre otros temas.

¿Cuál sería entonces el gran aporte de las competencias a los procesos de formación?. Definitivamente podría considerarse éste un modelo de formación integral en el que la respuesta al "¿para qué?" está siempre presente. Un modelo que obliga a cuestionarse alrededor de la pertinencia de los procesos educativos, invita a la educación a repensar al sujeto de aprendizaje como un agente transformador de la realidad, convoca al cuerpo docente a una reflexión y los llama a adaptarse a sus estudiantes, a sus procesos intelectivos, a sus preconceptos derivados de la experiencia y a sus aptitudes; y no pretender, aun cuando pareciera mas sencillo, que los estudiantes se adapten a sus docentes, pues finalmente son ellos los facilitadores.

La implementación de la formación por competencias demanda una transformación radical, mas no inmediata, de todo un paradigma educativo, implica cambios en la manera de hacer docencia, en la organización del sistema educativo, en la reflexión pedagógica y sobre todo de los esquemas de formación tan arraigados por la tradición.

El desarrollo de las competencias, al requerir de aprendizajes significativos, implica a los docentes abordar los procesos cognitivos e intelectivos de manera individual dentro del proceso de formación del estudiante, sin ello no se podrían lograr los niveles de comprensión que el estudiante necesita de los procesos que se dan dentro del aprendizaje.

Ante las diferentes perspectivas, el abordaje del contexto se constituye en un punto de discusión, pues si el contexto se aborda como saber disciplinar la evaluación se realiza en el marco de las disciplinas, como lo hace el ICFES. Por otra parte el contexto asumido como el entorno cultural, donde se da la puesta en escena del sujeto, no ofrece un panorama tan claro en el momento de determinar un esquema de evaluación debido a la complejidad misma de los contextos.

En ese sentido, si la formación por competencias aborda la dimensión del ser en el proceso de aprendizaje, en una evaluación por competencias cognitivas como la que realiza el ICFES ¿cómo se refleja la dimensión del ser en dicha evaluación?. Si la evaluación por competencias es una evaluación de procesos y no únicamente de resultados ¿no se constituyen los ECAES en una evaluación de resultados, tal como lo hace un modelo tradicional?.

Todas ellas son preguntas que solo se resolverán cuando se llegue a consensos conceptuales, pues mientras conceptos como el de competencias y contexto sigan siendo tan divergentes, en la implementación de la formación por competencias, aun cuando se mejoren algunos procesos educativos, 
seguirán reluciendo algunas incoherencias que constituirán un obstáculo para aquellos que pretendan abordarlas, y serán objeto de un extenso debate que apenas comienza.

\section{REFERENCIAS BIBLIOGRÁFICAS}

Bogoya, D., Torrado, Maria C. et al. Competencias Y Proyectos Pedagógicos. Capítulo: Educar para el desarrollo de las competencias: Una propuesta para reflexionar. Santa Fe de Bogotá. Universidad Nacional de Colombia. Mayo 2000

Chávez U. Las Competencias en la Educación para el trabajo. Seminario sobre Formación Profesional y Empleo. México D.F., 1998

DíAZ, F y HeRnÁNDEZ, G. Estrategias docentes para un aprendizaje significativo. México. Editorial Mc Graw Hill. 1998.

GÓMEZ E., Jairo. Lineamientos pedagógicos para una educación por competencias. Capítulo del libro: El concepto de competencia II. Una mirada interdisciplinar. Santa fe de Bogotá. Sociedad Colombiana de Pedagogía. 2002.

IAFRANCESCO V. Giovanni. Evaluación integral de aprendizajes. Taller. Universidad de Antioquia. Abril 29 y 30 de 2004.

ICFES. Nuevo examen de estado, Propuesta General. Santa Fe de Bogotá: ICFES, 1999.

MARín A., Luis. Competencias: "Saber hacer", ¿en cuál contexto?. Capítulo del libro: El concepto de competencia II. Una mirada interdisciplinar. Santa fe de Bogotá. Sociedad Colombiana de Pedagogía. 2002.

Segura, S. y Bejarano, A. Modelo Pedagógico de la Educación a Distancia Apoyada en las Tecnologías de la Información y la Comunicación en la Corporación Universitaria Autónoma de Occidente - CUAO. Memorias: Encuentro educación a distancia y entornos virtuales en la educación superior calidad, acreditación, experiencias y retos. Santiago de Cali, Mayo 7,8,9 de 2003.

TRUJILLO S., Fernando. Objetivos en la enseñanza de lenguas extranjeras: De la competencia lingüística a la competencia intercultural. Comunicación presentada en el Congreso Nacional "Inmigración, Convivencia e Interculturalidad", organizado en Ceuta por el Instituto de Estudios Ceutíes (Noviembre, 2001). 


\title{
Contactar
}

Revista lberoamericana de Educación

\author{
Principal OEI
}

